\title{
Information entropy of Gegenbauer polynomials of integer parameter
}

\author{
Julio I. de Vicente ${ }^{1}$, Silvia Gandy ${ }^{2}$ and Jorge Sánchez-Ruiz ${ }^{1,3}$ \\ 1 Departamento de Matemáticas, Universidad Carlos III de Madrid, \\ Avda. de la Universidad 30, E-28911 Leganés, Madrid, Spain \\ ${ }^{2}$ Fakultät für Mathematik, Technische Universität München, \\ Boltzmannstr. 3, D-85747 Garching b. München, Germany \\ ${ }^{3}$ Instituto Carlos I de Física Teórica y Computacional, Universidad de Granada, \\ E-18071 Granada, Spain \\ E-mail: jdvicent@math.uc3m.es, gandy@in.tum.de, jsanchez@math.uc3m.es
}

\begin{abstract}
The position and momentum information entropies of $D$-dimensional quantum systems with central potentials, such as the isotropic harmonic oscillator and the hydrogen atom, depend on the entropies of the (hyper)spherical harmonics. In turn, these entropies are expressed in terms of the entropies of the Gegenbauer (ultraspherical) polynomials $C_{n}^{(\lambda)}(x)$, the parameter $\lambda$ being either an integer or a half-integer number. Up to now, however, the exact analytical expression of the entropy of Gegenbauer polynomials of arbitrary degree $n$ has only been obtained for the particular values of the parameter $\lambda=0,1,2$. Here we present a novel approach to the evaluation of the information entropy of Gegenbauer polynomials, which makes use of trigonometric representations for these polynomials and complex integration techniques. Using this method, we are able to find the analytical expression of the entropy for arbitrary values of both $n$ and $\lambda \in \mathbb{N}$.
\end{abstract}

AMS classification scheme numbers: 30E20, 33B10, 33C45, 33F10, 42C05, 81Q99, $94 \mathrm{~A} 17$

PACS numbers: 03.67.-a, 02.30.Gp

Submitted to: J. Phys. A: Math. Gen. 


\section{Introduction}

According to Shannon's information theory [1, the only rigorous measure of the uncertainty or lack of information associated to a continuous random variable $X$ with density function $\rho(x), x \in \mathbb{R}^{D}$, is the entropy

$$
H(X)=-\int \rho(x) \log \rho(x) \mathrm{d} x .
$$

In particular, when $\rho(x)$ is the single-particle probability density for position of a quantum system, $H(X)$ is the only rigorous measure of the uncertainty in the localization of the particle in position space. The momentum entropy $H(P)$ can be defined likewise from the single-particle density of momentum $\gamma(p)$. In the simplest case of a single-particle system described in position space by the wave function $\psi(x)$, we have that $\rho(x)=|\psi(x)|^{2}$ and $\gamma(p)=|\phi(p)|^{2}$, where the wave function in momentum space $\phi(p)$ is the Fourier transform of $\psi(x)$. The sharp inequality [2, 3]

$$
H(X)+H(P) \geq D(1+\log \pi)
$$

places a nontrivial lower bound on the sum of the uncertainties in position and momentum, so it provides a quantitative formulation of the position-momentum uncertainty principle. Using the variational inequality that relates information entropy and standard deviation for an arbitrary $D$-dimensional random variable [1, 3],

$$
H(A) \leq \frac{D}{2}\left(1+\log \frac{2 \pi(\Delta A)^{2}}{D}\right)
$$

the entropic uncertainty relation (2) leads to the well-known Heisenberg uncertainty relation

$$
\Delta X \Delta P \geq \frac{D}{2}
$$

which proves the former to be stronger than the latter.

For many important quantum systems, such as $D$-dimensional harmonic oscillator

and hydrogen atom, the calculation of position and momentum information entropies involves the evaluation of integrals of the form

$$
E\left(p_{n}\right)=-\int_{a}^{b}\left(p_{n}(x)\right)^{2} \log \left(p_{n}(x)\right)^{2} \omega(x) \mathrm{d} x,
$$

where $\left\{p_{n}(x)\right\}$ denotes a polynomial sequence $\left(\operatorname{deg} p_{n}(x)=n\right)$ orthogonal on $[a, b] \subseteq \mathbb{R}$ with respect to the weight function $\omega(x)$. During the last decade there has been an intense activity in the study of these integrals, motivated not only by their relevance to quantum physics but also by their close relationship to other interesting mathematical objects, such as the $L^{p}$-norms or the logarithmic potentials of the polynomials $p_{n}(x)$. A survey on the state-of-the-art in this field up to year 2001 can be found in [4].

The calculation of the entropic integrals $E\left(p_{n}\right)$ is generally a very difficult task, and in most cases only asymptotic results for large values of $n$ are known 44. In fact, since all the zeros of $p_{n}$ are simple and belong to $(a, b)$, when $n$ is not very small even a numerical computation of $E\left(p_{n}\right)$ poses serious difficulties due to the strongly oscillatory 
behaviour of the integrand in (5). In this respect it is worth mentioning Ref. [5], which presents an efficient algorithm for the numerical evaluation of $E\left(p_{n}\right)$ in the case when the interval $(a, b)$ is finite.

Closed analytical formulas for $E\left(p_{n}\right)$ are only known for a few particular cases of the Gegenbauer or ultraspherical polynomials $C_{n}^{(\lambda)}$. We recall that these polynomials are defined as (see, e.g., [6, Sec. 4.7])

$$
C_{n}^{(\lambda)}(x)=\frac{(2 \lambda)_{n}}{\left(\lambda+\frac{1}{2}\right)_{n}} P_{n}^{\left(\lambda-\frac{1}{2}, \lambda-\frac{1}{2}\right)}(x),
$$

where $(a)_{n}=\Gamma(a+n) / \Gamma(a)$ denotes the Pochhammer symbol and $P_{n}^{(\alpha, \beta)}(x)$ are Jacobi polynomials,

$$
P_{n}^{(\alpha, \beta)}(x)=\frac{(\alpha+1)_{n}}{n !}{ }_{2} F_{1}\left(\begin{array}{c|c}
-n, n+\alpha+\beta+1 \\
\alpha+1
\end{array} \mid \frac{1-x}{2}\right) .
$$

For $\lambda>-\frac{1}{2}$, Gegenbauer polynomials form an orthogonal sequence on the interval $[-1,1]$ with respect to the weight function $w_{\lambda}(x)=\left(1-x^{2}\right)^{\lambda-\frac{1}{2}}$,

$$
\int_{-1}^{1} C_{n}^{(\lambda)}(x) C_{m}^{(\lambda)}(x)\left(1-x^{2}\right)^{\lambda-\frac{1}{2}} \mathrm{~d} x=\frac{2^{1-2 \lambda} \pi \Gamma(n+2 \lambda)}{(n+\lambda) n ![\Gamma(\lambda)]^{2}} \delta_{n, m} .
$$

The information entropies of Gegenbauer polynomials, on which we focus in the present paper, are thus given by

$$
E\left(C_{n}^{(\lambda)}\right)=-\int_{-1}^{1}\left(C_{n}^{(\lambda)}(x)\right)^{2} \log \left(C_{n}^{(\lambda)}(x)\right)^{2}\left(1-x^{2}\right)^{\lambda-\frac{1}{2}} \mathrm{~d} x .
$$

The integrals $E\left(C_{n}^{(\lambda)}\right)$ are especially relevant in the case when $\lambda$ is a non-negative integer or half-integer number, due to the relationship between the corresponding Gegenbauer polynomials and (hyper)spherical harmonics. As a consequence, these integrals appear in the calculation of the angular component of information entropies in both position and momentum space for any $D$-dimensional $(D \geq 2)$ quantummechanical system with a central potential, such as the isotropic harmonic oscillator or the hydrogen atom (radially symmetric Coulomb potential) [4, 7, 8, 9]. They also control the radial component of the information entropy in momentum space for the $D$-dimensional hydrogen atom [4, 7, 8].

Instead of using the standard definition of Gegenbauer polynomials, it is often more convenient to work with the polynomials

$$
\widehat{C}_{n}^{(\lambda)}(x)=\left(\frac{(n+\lambda) n !}{\lambda(2 \lambda)_{n}}\right)^{\frac{1}{2}} C_{n}^{(\lambda)}(x),
$$

which are orthonormal on $[-1,1]$ with respect to the probability density

$$
\widehat{w}_{\lambda}(x)=\frac{\Gamma(\lambda+1)}{\sqrt{\pi} \Gamma\left(\lambda+\frac{1}{2}\right)}\left(1-x^{2}\right)^{\lambda-\frac{1}{2}} .
$$

The corresponding entropies,

$$
E\left(\widehat{C}_{n}^{(\lambda)}\right)=-\int_{-1}^{1}\left[\widehat{C}_{n}^{(\lambda)}(x)\right]^{2} \log \left[\widehat{C}_{n}^{(\lambda)}(x)\right]^{2} \widehat{w}_{\lambda}(x) \mathrm{d} x,
$$


are related to $E\left(C_{n}^{(\lambda)}\right)$ by the formula

$$
E\left(\widehat{C}_{n}^{(\lambda)}\right)=\log \left(\frac{\lambda(2 \lambda)_{n}}{(n+\lambda) n !}\right)+\frac{\Gamma(\lambda)(n+\lambda) n !}{\sqrt{\pi} \Gamma\left(\lambda+\frac{1}{2}\right)(2 \lambda)_{n}} E\left(C_{n}^{(\lambda)}\right),
$$

which readily follows from the previous definitions by taking into account the orthogonality relation (8) .

The simplest particular cases of Gegenbauer polynomials are the Chebyshev polynomials of the first and second kind,

$$
T_{n}(x)=\lim _{\lambda \rightarrow 0} \frac{n !}{(2 \lambda)_{n}} C_{n}^{(\lambda)}(x), \quad U_{n}(x)=C_{n}^{(1)}(x) .
$$

For both of these families, information entropies can be computed in closed analytical form, the results being [7, 8]

$$
\begin{aligned}
& E\left(\widehat{T}_{n}\right)= \begin{cases}0 & \text { if } n=0, \\
\log 2-1 & \text { if } n \neq 0,\end{cases} \\
& E\left(\widehat{U}_{n}\right)=-\frac{n}{n+1} .
\end{aligned}
$$

In the $\lambda=2$ case, it was first proved in [10] that

$$
E\left(\widehat{C}_{n}^{(2)}\right)=-\log \left(\frac{3(n+1)}{n+3}\right)-\frac{n\left(n^{2}+2 n-1\right)}{(n+1)(n+2)(n+3)}-\frac{2}{\sqrt{(n+1)^{3}(n+3)^{3}}} \frac{T_{n+2}^{\prime \prime \prime}(\xi)}{T_{n+2}^{\prime \prime}(\xi)}
$$

where

$$
\xi=\frac{n+2}{\sqrt{(n+1)(n+3)}},
$$

and this result was later simplified to [11

$E\left(\widehat{C}_{n}^{(2)}\right)=-\log \left(\frac{3(n+1)}{n+3}\right)-\frac{n^{3}-5 n^{2}-29 n-27}{(n+1)(n+2)(n+3)}-\frac{1}{n+2}\left(\frac{n+3}{n+1}\right)^{n+2}$.

In the same work [11], it was also obtained the following generalization of (17) to arbitrary integer values of the parameter, $\lambda=l \in \mathbb{N}$ :

$$
E\left(\widehat{C}_{n}^{(l)}\right)=-s_{n l}-r_{n l} \sum_{j=1}^{2 l-2}\left(1-\xi_{j}^{2}\right) \frac{H\left(\xi_{j}\right)}{P^{\prime}\left(\xi_{j}\right)} \frac{\widehat{C}_{n-1}^{(l+1)}\left(\xi_{j}\right)}{\widehat{C}_{n}^{(l)}\left(\xi_{j}\right)},
$$

where $s_{n l}$ and $r_{n l}$ are known constants depending only on $n$ and $l$, the auxiliary polynomials $P$ and $H$ are defined from the sequence $\left\{P_{k}\right\}$ (deg $P_{k}=k$ ) generated by the recurrence relation

$$
P_{k+1}(x)=(2 l-2 k-3) x P_{k}(x)-(n+k+1)(n+2 l-k-1)\left(1-x^{2}\right) P_{k-1}(x)
$$

from the initial values $P_{-1}(x)=0, P_{0}(x)=1$ through the formulas

$$
P(x)=P_{2 l-2}(x), \quad H(x)=\sum_{s=0}^{2 l-2}(-1)^{s} P_{s-1}(x) P_{2 l-s-3}(x),
$$


and $\xi_{j}(j=1,2, \ldots, 2 l-2)$ denote the zeros of $P$. The explicit expression of the polynomial $P$ was later found to be 12

$P(x)=\frac{(-1)^{l-1}(n+2 l-1) !}{(n+l) n !} \sum_{\mu=0}^{l-1} \frac{(1-l)_{\mu}(l)_{\mu}(1 / 2)_{\mu}}{(1-n-l)_{\mu}(1+n+l)_{\mu} \mu !}\left(1-x^{2}\right)^{l-1-\mu}$.

Regretfully, (20) is not easy to use in practice. Furthermore, it is not a completely analytical formula save for small values of $l$ since, as we readily see from (23) , the zeros $\xi_{j}$ of $P$ have to be determined numerically when $l \geq 6 \pm$.

As first pointed out in [13], the entropy of Chebyshev polynomials of the first and second kind can be easily computed by direct calculation of the corresponding integrals by using the well-known trigonometric representations

$$
T_{n}(\cos \theta)=\cos n \theta, \quad U_{n}(\cos \theta)=\frac{\sin (n+1) \theta}{\sin \theta},
$$

with $x=\cos \theta$. Motivated by this observation, in the present paper we aim at evaluating the entropic integral $E\left(C_{n}^{(\lambda)}\right)$ for general values of the parameter $\lambda$ using representations of the same kind for the Gegenbauer polynomials.

We begin by collecting, in Section 2, the trigonometric representations of Gegenbauer polynomials that will be used later on. Our approach is developed in Section 3, where we show that it enables us to find completely analytical expressions for $E\left(C_{n}^{(\lambda)}\right)$, in terms of finite sums, whenever $\lambda \in \mathbb{N}$. The new results obtained for the information entropy of Gegenbauer polynomials of integer parameter are summarized in Section 4. Finally, in Section 5 some concluding remarks are given and several open problems are pointed out.

\section{Trigonometric representations for Gegenbauer polynomials}

The most widely known trigonometric representation of the Gegenbauer polynomials is (see e.g. [14, p. 302])

$$
C_{n}^{(\lambda)}(\cos \theta)=\sum_{m=0}^{n} d_{m, n}^{(\lambda)} \mathrm{e}^{\mathrm{i}(n-2 m) \theta}=\sum_{m=0}^{n} d_{m, n}^{(\lambda)} \cos (n-2 m) \theta,
$$

where

$$
d_{m, n}^{(\lambda)}=\frac{(\lambda)_{m}(\lambda)_{n-m}}{m !(n-m) !} .
$$

Another representation, due to Szegö [6, 15], is

$$
C_{n}^{(\lambda)}(\cos \theta)=\frac{c_{n}^{(\lambda)}}{(\sin \theta)^{2 \lambda-1}} \sum_{\nu=0}^{\infty} \alpha_{\nu, n}^{(\lambda)} \sin (n+2 \nu+1) \theta, \quad \lambda>0, \lambda \notin \mathbb{N},
$$

where

$$
c_{n}^{(\lambda)}=\frac{2^{2-2 \lambda} \Gamma(n+2 \lambda)}{\Gamma(\lambda) \Gamma(n+\lambda+1)}, \quad \alpha_{\nu, n}^{(\lambda)}=\frac{(1-\lambda)_{\nu}(n+1)_{\nu}}{\nu !(n+\lambda+1)_{\nu}} .
$$

$\ddagger$ Likewise, the general expression of $E\left(C_{n}^{(\lambda)}\right)$ given in [9] is not completely analytical save for small values of $n$, since it is expressed in terms of the zeros of $C_{n}^{(\lambda)}(x)$. 
At first sight, this representation seems to be less useful than the previous one, because it contains infinitely many terms. Moreover, it is supposed not to hold when $\lambda \in \mathbb{N}$. However, it is not difficult to prove that the validity of (27) extends to the case when $\lambda$ is a positive integer.

Proposition 1 The Szegö representation (27) holds true when $\lambda \in \mathbb{N}$. In this case, it reads

$$
C_{n}^{(\lambda)}(\cos \theta)=\frac{c_{n}^{(\lambda)}}{(\sin \theta)^{2 \lambda-1}} \sum_{\nu=0}^{\lambda-1} \alpha_{\nu, n}^{(\lambda)} \sin (n+2 \nu+1) \theta
$$

Proof If $\lambda \in \mathbb{N}$ then $\alpha_{\nu, n}^{(\lambda)}=0$ when $\nu \geq \lambda$, so that (27) reduces to (29). We will prove this equality by induction on $\lambda$. When $\lambda=1(29)$ is obviously true since it reduces to the second equation in (24), the well-known trigonometric representation for the Chebyshev polynomials of the second kind. Now, assume that the result holds for $\lambda=m-1$ $(m \in \mathbb{N})$. We take advantage of the following recurrence relation for the Gegenbauer polynomials [6, Eq. (4.7.27)],

$$
n C_{n}^{(\lambda)}(x)=(2 \lambda+n-1) x C_{n-1}^{(\lambda)}(x)-2 \lambda\left(1-x^{2}\right) C_{n-2}^{(\lambda+1)}(x),
$$

which in trigonometric form $(x=\cos \theta)$ can be restated as

$$
C_{n}^{(\lambda)}(\cos \theta)=\frac{1}{2(\lambda-1) \sin ^{2} \theta}\left[(2 \lambda+n-1) \cos \theta C_{n+1}^{(\lambda-1)}(\cos \theta)-(n+2) C_{n+2}^{(\lambda-1)}(\cos \theta)\right] \cdot(31
$$

Using this formula for $\lambda=m$ and substituting (29) on the right-hand-side we arrive at

$$
\begin{aligned}
C_{n}^{(m)}(\cos \theta)= & \frac{2 c_{n}^{(m)}}{(\sin \theta)^{2 m-1}}\left[\cos \theta \sum_{\nu=0}^{m-2} \alpha_{\nu, n+1}^{(m-1)} \sin (n+2 \nu+2) \theta\right. \\
& \left.-\frac{n+2}{n+m+1} \sum_{\nu=0}^{m-2} \alpha_{\nu, n+2}^{(m-1)} \sin (n+2 \nu+3) \theta\right] \\
= & \frac{c_{n}^{(m)}}{(\sin \theta)^{2 m-1}}\left[\sum_{\nu=0}^{m-2} \alpha_{\nu, n+1}^{(m-1)} \sin (n+2 \nu+3) \theta+\sum_{\nu=0}^{m-2} \alpha_{\nu, n+1}^{(m-1)} \sin (n+2 \nu+1) \theta\right. \\
& \left.-\frac{2(n+2)}{n+m+1} \sum_{\nu=0}^{m-2} \alpha_{\nu, n+2}^{(m-1)} \sin (n+2 \nu+3) \theta\right] \\
= & \frac{c_{n}^{(m)}}{(\sin \theta)^{2 m-1}} \sum_{\nu=0}^{m-1}\left(\alpha_{\nu-1, n+1}^{(m-1)}+\alpha_{\nu, n+1}^{(m-1)}-\frac{2(n+2)}{n+m+1} \alpha_{\nu-1, n+2}^{(m-1)}\right) \\
& \times \sin (n+2 \nu+1) \theta
\end{aligned}
$$

where in the last step we have used that $\alpha_{m-1, n+1}^{(m-1)}=0$ and $\alpha_{\nu, n+1}^{(m-1)}=0$ whenever $\nu<0$. A straightforward calculation shows that

$$
\alpha_{\nu-1, n+1}^{(m-1)}+\alpha_{\nu, n+1}^{(m-1)}-\frac{2(n+2)}{n+m+1} \alpha_{\nu-1, n+2}^{(m-1)}=\alpha_{\nu, n}^{(m)},
$$

and (29) is thus proved to hold also for $\lambda=m$. 
The fact that the sum in (27) terminates after a finite number of terms when $\lambda \in \mathbb{N}$ suggests that Szegö's representation may be useful to evaluate the entropy of Gegenbauer polynomials of integer parameter. Accordingly, in what follows we shall assume that $\lambda \in \mathbb{N}$ unless otherwise indicated.

\section{Evaluation of the entropic integral}

With the change of variable $x=\cos \theta$, the integral (9) takes the form

$$
E\left(C_{n}^{(\lambda)}\right)=-\int_{0}^{\pi}\left(C_{n}^{(\lambda)}(\cos \theta)\right)^{2} \log \left(C_{n}^{(\lambda)}(\cos \theta)\right)^{2} \sin ^{2 \lambda} \theta \mathrm{d} \theta
$$

Using Szegö's representation (29) for one of the two Gegenbauer polynomials in $\left(C_{n}^{(\lambda)}(\cos \theta)\right)^{2},(34)$ can be rewritten as

$$
E\left(C_{n}^{(\lambda)}\right)=-\frac{1}{2} c_{n}^{(\lambda)} \sum_{\nu=0}^{\lambda-1} \alpha_{\nu, n}^{(\lambda)}\left(J_{\nu, n}^{(\lambda)}-J_{\nu+1, n}^{(\lambda)}\right)
$$

where

$$
J_{\nu, n}^{(\lambda)}:=\int_{0}^{\pi} C_{n}^{(\lambda)}(\cos \theta) \cos (n+2 \nu) \theta \log \left(C_{n}^{(\lambda)}(\cos \theta)\right)^{2} \mathrm{~d} \theta
$$

Now, using the standard representation (25) we have that

$C_{n}^{(\lambda)}(\cos \theta) \cos (n+2 \nu) \theta=\frac{1}{2} \sum_{m=0}^{n} d_{m, n}^{(\lambda)} \cos 2(m+\nu) \theta+\frac{1}{2} \sum_{m=0}^{n} d_{m, n}^{(\lambda)} \cos 2(n-m+\nu) \theta$.

Taking into account the symmetry property $d_{m, n}^{(\lambda)}=d_{n-m, n}^{(\lambda)}$, which readily follows from the explicit expression of the coefficients $d_{m, n}^{(\lambda)}$, the previous equation simplifies to

$$
C_{n}^{(\lambda)}(\cos \theta) \cos (n+2 \nu) \theta=\sum_{m=0}^{n} d_{m, n}^{(\lambda)} \cos 2(m+\nu) \theta
$$

so that

$$
J_{\nu, n}^{(\lambda)}=\sum_{m=0}^{n} d_{m, n}^{(\lambda)} \int_{0}^{\pi} \cos 2(m+\nu) \theta \log \left(C_{n}^{(\lambda)}(\cos \theta)\right)^{2} \mathrm{~d} \theta
$$

Defining the integrals

$$
I_{m, n}^{(\lambda)}:=\int_{0}^{\pi} \cos (2 m \theta) \log \left(C_{n}^{(\lambda)}(\cos \theta)\right)^{2} \mathrm{~d} \theta,
$$

from (35) and (39) we find that $E\left(C_{n}^{(\lambda)}\right)$ is given by

$$
E\left(C_{n}^{(\lambda)}\right)=-\frac{1}{2} c_{n}^{(\lambda)} \sum_{\nu=0}^{\lambda-1} \alpha_{\nu, n}^{(\lambda)} \sum_{m=0}^{n} d_{m, n}^{(\lambda)}\left(I_{\nu+m, n}^{(\lambda)}-I_{\nu+1+m, n}^{(\lambda)}\right) .
$$

An alternative expression for the entropic integral $E\left(C_{n}^{(\lambda)}\right)$ which turns out to be more convenient in practice can be obtained by noticing that

$$
\begin{aligned}
E\left(C_{n}^{(\lambda)}\right) & =-\frac{1}{2} c_{n}^{(\lambda)} \sum_{\nu=0}^{\lambda-1} \alpha_{\nu, n}^{(\lambda)}\left\{\sum_{m=0}^{n} d_{m, n}^{(\lambda)} I_{\nu+m, n}^{(\lambda)}-\sum_{m=1}^{n+1} d_{m-1, n}^{(\lambda)} I_{\nu+m, n}^{(\lambda)}\right\} \\
& =-\frac{1}{2} c_{n}^{(\lambda)} \sum_{\nu=0}^{\lambda-1} \alpha_{\nu, n}^{(\lambda)}\left\{\sum_{m=1}^{n}\left(d_{m, n}^{(\lambda)}-d_{m-1, n}^{(\lambda)}\right) I_{\nu+m, n}^{(\lambda)}+d_{0, n}^{(\lambda)} I_{\nu, n}^{(\lambda)}-d_{n, n}^{(\lambda)} I_{\nu+n+1, n}^{(\lambda)}\right\} .
\end{aligned}
$$


According to (26) $), d_{m, n}^{(\lambda)}=0$ when $-\lambda<m<0$ or $n<m<n+\lambda$. Let us restrict initially to the case when $\lambda \neq 1$, so that $d_{-1, n}^{(\lambda)}=0$ and $d_{n+1, n}^{(\lambda)}=0$. This allows us to write the previous formula in the more compact form

$$
\begin{aligned}
E\left(C_{n}^{(\lambda)}\right) & =-\frac{1}{2} c_{n}^{(\lambda)} \sum_{\nu=0}^{\lambda-1} \sum_{m=0}^{n+1} \alpha_{\nu, n}^{(\lambda)}\left(d_{m, n}^{(\lambda)}-d_{m-1, n}^{(\lambda)}\right) I_{\nu+m, n}^{(\lambda)} \\
& =-\frac{1}{2} c_{n}^{(\lambda)} \sum_{\nu=0}^{\lambda-1} \sum_{m=\nu}^{n+1+\nu} \alpha_{\nu, n}^{(\lambda)}\left(d_{m-\nu, n}^{(\lambda)}-d_{m-\nu-1, n}^{(\lambda)}\right) I_{m, n}^{(\lambda)} .
\end{aligned}
$$

Using again that $d_{m, n}^{(\lambda)}=0$ when $-\lambda<m<0$ as well as when $n<m<n+\lambda$, we can extend the lower and upper limits in the inner summation to 1 and $n+\lambda-1$, respectively, provided that the terms $m=0$ and $m=n+\lambda$ are treated separately. Thus we find that

$E\left(C_{n}^{(\lambda)}\right)=-\frac{1}{2} c_{n}^{(\lambda)}\left(\alpha_{0, n}^{(\lambda)} d_{0, n}^{(\lambda)} I_{0, n}^{(\lambda)}-\alpha_{\lambda-1, n}^{(\lambda)} d_{n, n}^{(\lambda)} I_{n+\lambda, n}^{(\lambda)}+\sum_{m=1}^{n+\lambda-1} \beta_{m, n}^{(\lambda)} I_{m, n}^{(\lambda)}\right)$,

where

$$
\beta_{m, n}^{(\lambda)}=\sum_{\nu=0}^{\lambda-1} \alpha_{\nu, n}^{(\lambda)}\left(d_{m-\nu, n}^{(\lambda)}-d_{m-\nu-1, n}^{(\lambda)}\right) .
$$

It can be seen that (44) also holds when $\lambda=1$ by noting that in this case its right-hand side coincides with that of (42).

In order to apply (44), we need to evaluate the integrals $I_{m, n}^{(\lambda)}$ with $0 \leq m \leq n+\lambda$. This goal can be achieved by means of complex integration techniques, which enable us to obtain the following result.

Theorem 1 For $\lambda \in \mathbb{N}$,

$$
I_{0, n}^{(\lambda)}=2 \pi \log \left(\frac{(\lambda)_{n}}{n !}\right)
$$

and, when $m \geq 1$,

$$
I_{m, n}^{(\lambda)}=\frac{(2 \lambda-1) \pi}{m}+\left.\frac{\pi}{(2 m) !} \frac{d^{2 m}}{d z^{2 m}}\left(\log \sum_{\nu=0}^{\lambda-1} \alpha_{\nu, n}^{(\lambda)}\left(z^{2 n+2 \lambda+2 \nu}-z^{2 \lambda-2 \nu-2}\right)\right)\right|_{z=0} .
$$

Proof Taking into account that $C_{n}^{(\lambda)}(-x)=(-1)^{n} C_{n}^{(\lambda)}(x)$, (40) can be written as

$$
\begin{aligned}
I_{m, n}^{(\lambda)} & =\frac{1}{2} \int_{0}^{2 \pi} \cos (2 m \theta) \log \left|C_{n}^{(\lambda)}(\cos \theta)\right|^{2} \mathrm{~d} \theta \\
& =\int_{0}^{2 \pi} \cos (2 m \theta) \log \left|C_{n}^{(\lambda)}(\cos \theta)\right| \mathrm{d} \theta \\
& =\int_{0}^{2 \pi} \cos (2 m \theta) \log \left|\mathrm{e}^{\mathrm{i} n \theta} C_{n}^{(\lambda)}(\cos \theta)\right| \mathrm{d} \theta,
\end{aligned}
$$


where in the last step the factor $\mathrm{e}^{\mathrm{i} n \theta}$ has been introduced for later convenience. Using the Szegö representation (29) for the Gegenbauer polynomial $C_{n}^{(\lambda)}(\cos \theta)$, the previous equation reads

$$
I_{m, n}^{(\lambda)}=\int_{0}^{2 \pi} \cos (2 m \theta) \log \left|\frac{c_{n}^{(\lambda)} \mathrm{e}^{\mathrm{i} n \theta}}{\sin ^{2 \lambda-1} \theta} \sum_{\nu=0}^{\lambda-1} \alpha_{\nu, n}^{(\lambda)} \sin (n+2 \nu+1) \theta\right| \mathrm{d} \theta .
$$

We will compute the integral

$$
\mathcal{I}_{m, n}^{(\lambda)}=\int_{0}^{2 \pi} \cos (2 m \theta) \log \left(\frac{c_{n}^{(\lambda)} \mathrm{e}^{\mathrm{i} n \theta}}{\sin ^{2 \lambda-1} \theta} \sum_{\nu=0}^{\lambda-1} \alpha_{\nu, n}^{(\lambda)} \sin (n+2 \nu+1) \theta\right) \mathrm{d} \theta,
$$

whose real part equals $I_{m, n}^{(\lambda)}$. Introducing the change of variable $z=\exp (\mathrm{i} \theta)$ we arrive at

$$
\mathcal{I}_{m, n}^{(\lambda)}=\frac{1}{2 \mathrm{i}} \oint_{|z|=1} \frac{z^{4 m}+1}{z^{2 m+1}} \log q(z) \mathrm{d} z
$$

where

$q(z)=z^{n} C_{n}^{(\lambda)}\left(\frac{z+z^{-1}}{2}\right)=c_{n}^{(\lambda)} 2^{2 \lambda-2}(-1)^{\lambda} \frac{\sum_{\nu=0}^{\lambda-1} \alpha_{\nu, n}^{(\lambda)}\left(z^{2 n+2 \lambda+2 \nu}-z^{2 \lambda-2 \nu-2}\right)}{\left(1-z^{2}\right)^{2 \lambda-1}}$.

The singularities of the integrand are $z=0$, which is a pole of order $2 m+1$, and all the zeros of $q(z)$, which are branch points. If $\left\{x_{n, j}\right\}_{j=1}^{n}$ denote the zeros of $C_{n}^{(\lambda)}(x)$, which are known to be simple, real and located in $(-1,1)$, then the zeros $\left\{z_{n, j}\right\}_{j=1}^{2 n}$ of the function $q(z)$ are

$$
z_{n, j+\frac{n}{2} \mp \frac{n}{2}}=\exp \left(\mathrm{i} \arccos x_{n, j}\right)=x_{n, j} \pm \mathrm{i} \sqrt{1-x_{n, j}^{2}}, \quad j=1,2, \ldots, n .
$$

This means that the $\left\{z_{n, j}\right\}_{j=1}^{2 n}$ are all located on the unit circle, which can also be seen from the fact that $z=\exp (\mathrm{i} \arccos x)$ maps $(-1,1)$ onto the unit circle. Therefore, the integrand of (51) has $2 n$ branch points located on the contour of integration. To avoid this difficulty we consider the same integral along the slightly different contour $\Gamma$ (see Figure 1), which is also closed. Notice that the logarithmic branches can be chosen to go from the branch points to the exterior of the unit disk, so that $\Gamma$ does not cross them. Since the only singularity inside $\Gamma$ is $z=0$ we now have

$$
\oint_{\Gamma} \frac{z^{4 m}+1}{z^{2 m+1}} \log q(z) \mathrm{d} z=2 \pi \mathrm{i} \operatorname{Res}\left(\frac{z^{4 m}+1}{z^{2 m+1}} \log q(z), z=0\right) .
$$

The integral along $\Gamma$ can be decomposed as

$$
\oint_{\Gamma} \frac{z^{4 m}+1}{z^{2 m+1}} \log q(z) \mathrm{d} z=\sum_{j=1}^{2 n}\left(\int_{\epsilon_{j}} \frac{z^{4 m}+1}{z^{2 m+1}} \log q(z) \mathrm{d} z+\int_{\gamma_{j}} \frac{z^{4 m}+1}{z^{2 m+1}} \log q(z) \mathrm{d} z\right),
$$

where $\epsilon_{j}$ denotes the arc of circumference of radius $\varepsilon$ that surrounds the branch point $z_{n, j}$ and $\gamma_{j}$ denotes the arc on the unit circle that connects $\epsilon_{j}$ and $\epsilon_{j+1}\left(\gamma_{2 n}\right.$ connects $\epsilon_{2 n}$ and $\left.\epsilon_{1}\right)$. Parameterizing $z=z_{n, j}+\varepsilon \mathrm{e}^{\mathrm{i} \theta}$ we find that

$$
\int_{\epsilon_{j}} \frac{z^{4 m}+1}{z^{2 m+1}} \log q(z) \mathrm{d} z=\int_{\epsilon_{j}} \frac{\left(z_{n, j}+\varepsilon \mathrm{e}^{\mathrm{i} \theta}\right)^{4 m}+1}{\left(z_{n, j}+\varepsilon \mathrm{e}^{\mathrm{i} \theta}\right)^{2 m+1}} \log q\left(z_{n, j}+\varepsilon \mathrm{e}^{\mathrm{i} \theta}\right) \mathrm{i} \varepsilon \mathrm{e}^{\mathrm{i} \theta} d \theta \underset{\varepsilon \rightarrow 0}{\longrightarrow} 0,
$$




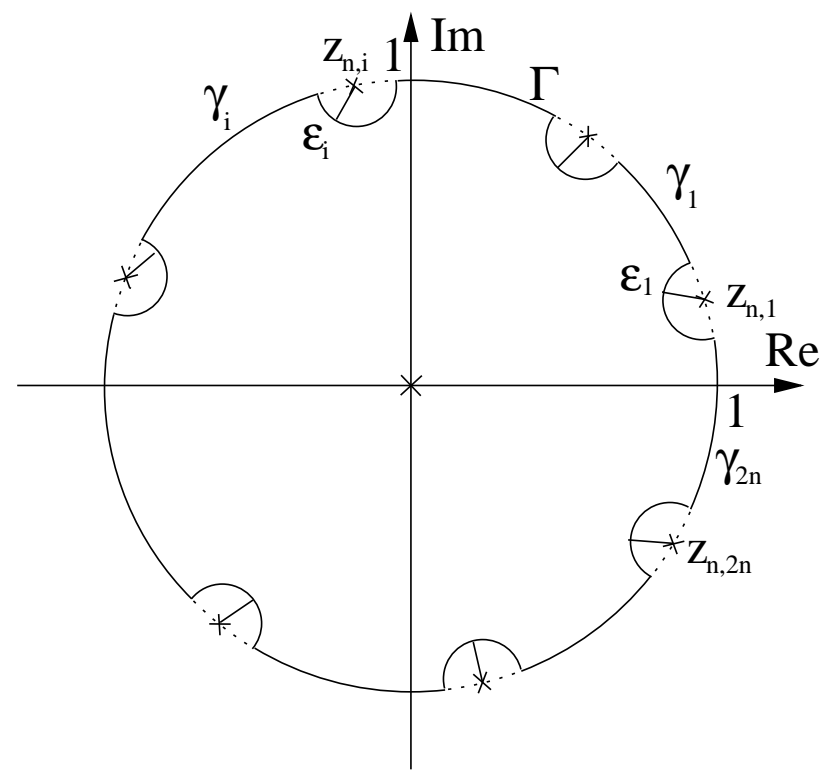

Figure 1. Integration contour used to avoid the branch points on the unit circle.

where we have used that $x \log x \rightarrow 0$ as $x \rightarrow 0$. Thus, taking the limit $\varepsilon \rightarrow 0$ in (55) we conclude that

$$
\oint_{|z|=1} \frac{z^{4 m}+1}{z^{2 m+1}} \log q(z) \mathrm{d} z=\oint_{\Gamma} \frac{z^{4 m}+1}{z^{2 m+1}} \log q(z) \mathrm{d} z .
$$

Taking into account that the residue of a meromorphic function $h(z)$ in a pole $z_{0}$ of order $2 m+1$ is given by

$$
\operatorname{Res}\left(h(z), z=z_{0}\right)=\left.\frac{1}{(2 m) !} \frac{d^{2 m}}{d z^{2 m}}\left(\left(z-z_{0}\right)^{2 m+1} h(z)\right)\right|_{z=z_{0}},
$$

use of (54) and (57) into (51) leads to

$$
\mathcal{I}_{m, n}^{(\lambda)}=\left.\frac{\pi}{(2 m) !} \frac{d^{2 m}}{d z^{2 m}}\left[\left(z^{4 m}+1\right) \log q(z)\right]\right|_{z=0} .
$$

In the case $m=0$, the previous equation reduces to

$$
\mathcal{I}_{0, n}^{(\lambda)}=2 \pi \log q(0)=2 \pi \log \left(c_{n}^{(\lambda)} 2^{2 \lambda-2}(-1)^{\lambda+1} \alpha_{\lambda-1, n}^{(\lambda)}\right),
$$

so that

$$
I_{0, n}^{(\lambda)}=2 \pi \log \left|c_{n}^{(\lambda)} 2^{2 \lambda-2} \alpha_{\lambda-1, n}^{(\lambda)}\right|=2 \pi \log \left(\frac{(\lambda)_{n}}{n !}\right),
$$

which proves the first part of the theorem \&]. On the other hand, if $m \geq 1$ then we readily see from (59) that $\mathcal{I}_{m, n}^{(\lambda)} \in \mathbb{R}$, so $I_{m, n}^{(\lambda)}=\mathcal{I}_{m, n}^{(\lambda)}$. Furthermore, in this case the factor $\left(z^{4 m}+1\right)$ in the right-hand side of (59) can be omitted, since at $z=0$ its value equals unity while all its derivatives do vanish. We thus find that

$$
I_{m, n}^{(\lambda)}=\left.\frac{\pi}{(2 m) !} \frac{d^{2 m}}{d z^{2 m}}\left[\log \left(\frac{\sum_{\nu=0}^{\lambda-1} \alpha_{\nu, n}^{(\lambda)}\left(z^{2 n+2 \lambda+2 \nu}-z^{2 \lambda-2 \nu-2}\right)}{\left(1-z^{2}\right)^{2 \lambda-1}}\right)\right]\right|_{z=0}
$$

$\S$ This part can also be proved using the mean value theorem for harmonic functions (cf. [7, Sec. VI]). 
and (47) follows on noting that

$$
\left.\frac{d^{2 m}}{d z^{2 m}}\left(\log \left(1-z^{2}\right)\right)\right|_{z=0}=\left.\frac{d^{2 m}}{d z^{2 m}}\left(-\sum_{k=1}^{\infty} \frac{z^{2 k}}{k}\right)\right|_{z=0}=-\frac{(2 m) !}{m}
$$

when $m \neq 0$.

The fact that the Szegö representation (29) has a finite number of terms plays an essential role in the proof of Theorem 1. Although we are mainly interested in evaluating the integrals $I_{m, n}^{(\lambda)}$ when $\lambda \in \mathbb{N}$, it is worth pointing out that these integrals can be calculated in a similar way for all possible values of $\lambda$, provided that we use the standard trigonometric representation (25) instead of the Szegö representation for the Gegenbauer polynomial inside the logarithm. This generalization is contained in the next theorem.

Theorem 2 For $\lambda \in \mathbb{R}, \lambda>-\frac{1}{2}$,

$$
I_{0, n}^{(\lambda)}=2 \pi \log \left(\frac{(\lambda)_{n}}{n !}\right)
$$

and, when $m \geq 1$,

$$
I_{m, n}^{(\lambda)}=\left.\frac{\pi}{(2 m) !} \frac{d^{2 m}}{d z^{2 m}}\left(\log \sum_{j=0}^{n} d_{j, n}^{(\lambda)} z^{2 n-2 j}\right)\right|_{z=0} .
$$

Proof We proceed as in the proof of Theorem 1, but now we use the complex form of the standard trigonometric representation (25) for the Gegenbauer polynomial $C_{n}^{(\lambda)}(\cos \theta)$ in (48). Thus we arrive at

$$
I_{m, n}^{(\lambda)}=\left.\frac{\pi}{(2 m) !} \frac{d^{2 m}}{d z^{2 m}}\left[\left(z^{4 m}+1\right) \log \left(\sum_{j=0}^{n} d_{j, n}^{(\lambda)} z^{2 n-2 j}\right)\right]\right|_{z=0},
$$

from which (64) and (65) readily follow.

In order to carry out the sums in (44), the next step is to obtain closed formulas for the derivatives $I_{m, n}^{(\lambda)}$ with $1 \leq m \leq n+\lambda$. Despite its greater generality, Theorem 2 turns out to be less useful than Theorem 1, because (47) expresses the integrals in terms of the logarithm of a polynomial that has $2 \lambda$ terms, while in (65) they are given in terms of the logarithm of a polynomial with $n+1$ terms. As we shall see, the difficulty in obtaining a closed formula for the derivatives of such functions increases with the number of terms in the polynomial. Therefore, if we want an expression of $I_{m, n}^{(\lambda)}$ for a fixed value of $\lambda$ and any $n \in \mathbb{N}$ Theorem 1 is more helpful, particularly for small values of $\lambda$.

In the case $\lambda=1$ we readily notice from (47) that, if $1 \leq m \leq n+1$, then

$$
\begin{aligned}
I_{m, n}^{(1)} & =\frac{\pi}{m}+\left.\frac{\pi}{(2 m) !} \frac{d^{2 m}}{d z^{2 m}}\left(\log \left(1-z^{2 n+2}\right)\right)\right|_{z=0} \\
& =\frac{\pi}{m}+\left.\frac{\pi}{(2 m) !} \frac{d^{2 m}}{d z^{2 m}}\left(-\sum_{k=1}^{\infty} \frac{z^{(2 n+2) k}}{k}\right)\right|_{z=0} \\
& =\pi\left(\frac{1}{m}-\delta_{m, n+1}\right) .
\end{aligned}
$$


When $\lambda \geq 2$, the polynomial inside the logarithm has more terms and the above trick does not work. However, we can obtain closed formulas for the derivatives in (47) by means of Faà di Bruno's formula for the derivatives of the composition of two functions, which states that (see e.g. [16])

$$
\frac{d^{m} f(g(z))}{d z^{m}}=m ! \sum_{k=0}^{m} f^{(k)}(g(z)) \sum_{k_{1}, k_{2}, \ldots, k_{m}} \prod_{j=1}^{m} \frac{\left[g^{(j)}(z)\right]^{k_{j}}}{(j !)^{k_{j}} k_{j} !}
$$

where the inner summation is extended over all partitions satisfying

$$
k_{1}+k_{2}+\cdots+k_{m}=k, \quad k_{1}+2 k_{2}+\cdots+m k_{m}=m .
$$

This formula enables us to find explicit expressions for $I_{m, n}^{(\lambda)}$ with $\lambda \geq 2$, as stated in the following two propositions.

Proposition 2 In the case $\lambda=2$, when $1 \leq m \leq n+2$

$$
I_{m, n}^{(2)}=\frac{\pi}{m}\left[3-\left(\frac{n+3}{n+1}\right)^{m}\right]+\pi \frac{n+3}{n+1} \delta_{m, n+2} .
$$

Proof In this case, application of Faà di Bruno's formula (68) to the derivatives in (47) gives

$$
\begin{gathered}
\left.\frac{d^{2 m}}{d z^{2 m}}\left(\log \sum_{\nu=0}^{1} \alpha_{\nu, n}^{(2)}\left(z^{2 n+4+2 \nu}-z^{2-2 \nu}\right)\right)\right|_{z=0}=\left.(2 m) ! \sum_{k=1}^{2 m} \frac{d^{k}}{d z^{k}}(\log z)\right|_{z=-\alpha_{1, n}^{(2)}} \\
\times \sum_{k_{1}, k_{2}, \ldots, k_{2 m}} \prod_{j=1}^{2 m} \frac{\left[\left.\frac{d^{j}}{d z^{j}}\left(\sum_{\nu=0}^{1} \alpha_{\nu, n}^{(2)}\left(z^{2 n+4+2 \nu}-z^{2-2 \nu}\right)\right)\right|_{z=0}\right]^{k_{j}}}{(j !)^{k_{j}} k_{j} !} .
\end{gathered}
$$

On the one hand, for $k \geq 1$,

$$
\frac{d^{k}}{d z^{k}}(\log z)=\frac{(-1)^{k+1}(k-1) !}{z^{k}}
$$

so that

$$
\left.\frac{d^{k}}{d z^{k}}(\log z)\right|_{z=-\alpha_{1, n}^{(2)}}=-\frac{(k-1) !}{\left(\alpha_{1, n}^{(2)}\right)^{k}} .
$$

On the other hand, all the derivatives of the polynomial in (171) vanish at $z=0$ except when $j=2$ and $j=2 n+4$, so we must set $k_{j}=0$ if $j \neq 2$ and $j \neq 2 n+4$. Conditions (69) then read

$$
k_{2}+k_{2 n+4}=k, \quad 2 k_{2}+(2 n+4) k_{2 n+4}=2 m .
$$

Since $k_{2}$ and $k_{2 n+4}$ are non-negative integers, these equations only admit the solution $k_{2 n+4}=0, k_{2}=k=m$ when $m \leq n+1$, while in the case $m=n+2$ we have to add the solution $k_{2 n+4}=k=1, k_{2}=0$ to the previous one. Therefore, (71) simplifies to

$\left.\frac{d^{2 m}}{d z^{2 m}}\left(\log \sum_{\nu=0}^{1} \alpha_{\nu, n}^{(2)}\left(z^{2 n+4+2 \nu}-z^{2-2 \nu}\right)\right)\right|_{z=0}=-(2 m) !\left[\frac{1}{m}\left(-\frac{\alpha_{0, n}^{(2)}}{\alpha_{1, n}^{(2)}}\right)^{m}+\frac{\alpha_{0, n}^{(2)}}{\alpha_{1, n}^{(2)}} \delta_{m, n+2}\right]$

and the result follows using the second equation in (28).

$\|$ Notice that attending to (69) $k=0$ corresponds to $m=0$, so we can start the sum in $k$ from 1. 
Proposition 3 For any $\lambda \in \mathbb{N}, \lambda \geq 3$, when $1 \leq m \leq n+\lambda$

$$
\begin{aligned}
I_{m, n}^{(\lambda)}= & \frac{(2 \lambda-1) \pi}{m}-\pi \frac{\alpha_{0, n}^{(\lambda)}}{\alpha_{\lambda-1, n}^{(\lambda)}} \delta_{m, n+\lambda} \\
& -\pi\left(\frac{\alpha_{\lambda-3, n}^{(\lambda)}}{\alpha_{\lambda-2, n}^{(\lambda)}}\right)^{m} \sum_{k=1}^{m} \sum_{k_{6}=0}^{[m / 3]} \sum_{k_{8}=0}^{[m / 4]} \cdots \sum_{k_{2(\lambda-1)=0}}^{[m /(\lambda-1)]}\left(-\frac{\left(\alpha_{\lambda-2, n}^{(\lambda)}\right)^{2}}{\alpha_{\lambda-1, n}^{(\lambda)} \alpha_{\lambda-3, n}^{(\lambda)}}\right)^{k} \\
& \times \frac{(k-1) !}{\left(2 k-m+\sum_{r=1}^{\lambda-3} r k_{2 r+4}\right) !\left(m-k-\sum_{s=1}^{\lambda-3}(s+1) k_{2 s+4}\right) !} \\
& \times \prod_{j=3}^{\lambda-1} \frac{1}{\left(k_{2 j}\right) !}\left(-\frac{\alpha_{\lambda-1-j, n}^{(\lambda)}\left(\alpha_{\lambda-2, n}^{(\lambda)}\right)^{j-2}}{\left(\alpha_{\lambda-3, n}^{(\lambda)}\right)^{j-1}}\right)^{k_{2 j}}
\end{aligned}
$$

where in the upper limits of the summations over $k_{6}, k_{8}, \ldots, k_{2(\lambda-1)}$ the square brackets denote integer part of the expression within.

In particular, in the case $\lambda=3$, when $1 \leq m \leq n+3$

$$
I_{m, n}^{(3)}=\frac{\pi}{m}\left[5-2 \Re\left(f(n)^{m}\right)\right]-\pi \frac{(n+4)(n+5)}{(n+1)(n+2)} \delta_{m, n+3},
$$

where

$$
f(n)=\frac{(n+1)(n+5)+\mathrm{i} \sqrt{3(n+1)(n+5)}}{(n+1)(n+2)} .
$$

Proof In the general case $(\lambda \in \mathbb{N}, \lambda \geq 3)$, application of Faà di Bruno's formula (68) to the derivatives in (47) and use of (72) lead to

$$
\begin{aligned}
& \left.\frac{d^{2 m}}{d z^{2 m}}\left(\log \sum_{\nu=0}^{\lambda-1} \alpha_{\nu, n}^{(\lambda)}\left(z^{2 n+2 \lambda+2 \nu}-z^{2 \lambda-2 \nu-2}\right)\right)\right|_{z=0} \\
& =-(2 m) ! \sum_{k=1}^{2 m} \frac{(k-1) !}{\left(\alpha_{\lambda-1, n}^{(\lambda)}\right)^{k}} \sum_{k_{1}, k_{2}, \ldots, k_{2 m}} \prod_{j=1}^{2 m} \frac{\left[\left.\frac{d^{j}}{d z^{j}}\left(\sum_{\nu=0}^{\lambda-1} \alpha_{\nu, n}^{(\lambda)} z^{2 n+2 \lambda+2 \nu}\right)\right|_{z=0}\right]^{k_{j}}}{(j !)^{k_{j}} k_{j} !} \\
& -(2 m) ! \sum_{k=1}^{2 m} \frac{(k-1) !}{\left(\alpha_{\lambda-1, n}^{(\lambda)}\right)^{k}} \sum_{k_{1}, k_{2}, \ldots, k_{2 m}} \prod_{j=1}^{2 m} \frac{\left[\left.\frac{d^{j}}{d z^{j}}\left(-\sum_{\nu=0}^{\lambda-1} \alpha_{\nu, n}^{(\lambda)} z^{2 \lambda-2 \nu-2}\right)\right|_{z=0}\right]^{k_{j}}}{(j !)^{k_{j}} k_{j} !} .
\end{aligned}
$$

In the first term of the right-hand side all derivatives vanish at $z=0$ except when $j=2 n+2 \lambda$, so that $k_{j}=0$ whenever $j \neq 2 n+2 \lambda$ and conditions (69) simplify to

$$
k_{2 n+2 \lambda}=k, \quad(2 n+2 \lambda) k_{2 n+2 \lambda}=2 m,
$$

which only admit the solution $k_{2 n+2 \lambda}=k=1$ when $m=n+\lambda$. In the second term the derivatives that do not vanish are those with $j$ even, $2 \leq j \leq 2 \lambda-2$, so that conditions (69) now read

$$
\sum_{r=1}^{\lambda-1} k_{2 r}=k, \quad \sum_{s=1}^{\lambda-1} s k_{2 s}=m .
$$


Equation (79) thus reduces to

$$
\begin{gathered}
\left.\frac{d^{2 m}}{d z^{2 m}}\left(\log \sum_{\nu=0}^{\lambda-1} \alpha_{\nu, n}^{(\lambda)}\left(z^{2 n+2 \lambda+2 \nu}-z^{2 \lambda-2 \nu-2}\right)\right)\right|_{z=0}=-(2 n+2 \lambda) ! \frac{\alpha_{0, n}^{(\lambda)}}{\alpha_{\lambda-1, n}^{(\lambda)}} \delta_{m, n+\lambda} \\
-(2 m) ! \sum_{k=1}^{2 m} \frac{(k-1) !}{\left(\alpha_{\lambda-1, n}^{(\lambda)}\right)^{k}} \sum_{k_{2}, k_{4}, \ldots, k_{2(\lambda-1)}} \prod_{j=1}^{2 m} \frac{\left(-\alpha_{\lambda-1-j, n}^{(\lambda)}\right)^{k_{2 j}}}{\left(k_{2 j}\right) !} .
\end{gathered}
$$

Finally, we can further simplify the previous expression to obtain (76) by using conditions (81) to write $k_{2}$ and $k_{4}$ in terms of the remaining indices, i.e.

$$
\begin{aligned}
& k_{2}=k_{6}+2 k_{8}+\cdots+(\lambda-3) k_{2(\lambda-1)}+2 k-m, \\
& k_{4}=-2 k_{6}-3 k_{8}-\cdots-(\lambda-2) k_{2(\lambda-1)}+m-k .
\end{aligned}
$$

Notice that in (176) conditions (181) are guaranteed to hold because for the values of the indices that do not fulfill them we get the inverse of the factorial of a negative integer, which can be considered to be zero. We have changed the upper limit in the sum over $k$ from $2 m$ to $m$ because when $m+1 \leq k \leq 2 m$ conditions (81) are not fulfilled.

In the case $\lambda=3$, (176) reduces to

$I_{m, n}^{(3)}=\frac{5 \pi}{m}-\pi\left(\frac{\alpha_{0, n}^{(3)}}{\alpha_{1, n}^{(3)}}\right)^{m} \sum_{k=1}^{m} \frac{(k-1) !}{(2 k-m) !(m-k) !}\left(-\frac{\left(\alpha_{1, n}^{(3)}\right)^{2}}{\alpha_{2, n}^{(3)} \alpha_{0, n}^{(3)}}\right)^{k}-\pi \frac{\alpha_{0, n}^{(3)}}{\alpha_{2, n}^{(3)}} \delta_{m, n+3}$,

so we need to evaluate a sum of the form

$$
\sum_{k=1}^{m} \frac{(k-1) !}{(2 k-m) !(m-k) !} x^{k}=\sum_{j=0}^{m-1} \frac{(m-j-1) !}{j !(m-2 j) !} x^{m-j}=x^{m} \sum_{j=0}^{m-1}\left(\begin{array}{c}
m-j \\
j
\end{array}\right) \frac{\left(x^{-1}\right)^{j}}{m-j} .
$$

Using the summation formula [17, Eq. (5.75)]

$$
\sum_{j=0}^{m-1}\left(\begin{array}{c}
m-j \\
j
\end{array}\right) \frac{m}{m-j} z^{j}=\left(\frac{1+\sqrt{1+4 z}}{2}\right)^{m}+\left(\frac{1-\sqrt{1+4 z}}{2}\right)^{m}
$$

and the second equation in (28), we find that

$$
I_{m, n}^{(3)}=\frac{5 \pi}{m}-\frac{\pi}{m}\left[f(n)^{m}+f^{*}(n)^{m}\right]-\pi \frac{(n+4)(n+5)}{(n+1)(n+2)} \delta_{m, n+3},
$$

which is equivalent to (77).

\section{Results for the information entropy}

Equations (46), (67), (70) and (77) enable us to derive closed analytical formulas for $E\left(C_{n}^{(\lambda)}\right)$ when $\lambda=1,2,3$. For $\lambda=1$, after substitution of the corresponding values of the constants $d_{m, n}^{(\lambda)}, c_{n}^{(\lambda)}$ and $\alpha_{\nu, n}^{(\lambda)}$ (see (26) and (28) ), (44) reduces to

$$
E\left(C_{n}^{(1)}\right)=-\frac{1}{2}\left(I_{0, n}^{(1)}-I_{n+1, n}^{(1)}\right),
$$

which using (46) and (67) immediately leads to

$$
E\left(C_{n}^{(1)}\right)=E\left(U_{n}\right)=\frac{\pi}{2}\left(\frac{1}{n+1}-1\right) .
$$


When $\lambda=2$, (44) takes the form

$$
E\left(C_{n}^{(2)}\right)=-\frac{1}{8}\left((n+1)(n+3) I_{0, n}^{(2)}-(n+1)^{2} I_{n+2, n}^{(2)}-4 \sum_{m=1}^{n+1} m I_{m, n}^{(2)}\right)
$$

so using (46) and (70) together with the well-known formula for the sum of a geometric series,

$$
\sum_{m=1}^{n} x^{m}=\frac{x\left(1-x^{n}\right)}{1-x}
$$

we find that

$$
\begin{aligned}
E\left(C_{n}^{(2)}\right)=-\frac{\pi}{8} & \left(2(n+1)(n+3) \log (n+1)+\frac{n^{3}-5 n^{2}-29 n-27}{n+2}\right. \\
& \left.+\frac{(n+3)^{n+3}}{(n+2)(n+1)^{n+1}}\right) .
\end{aligned}
$$

Recalling (13), (89) and (92) are readily shown to be equivalent to (16) and (19), respectively.

In the case $\lambda=3$, (44) can be rewritten as

$$
\begin{aligned}
E\left(C_{n}^{(3)}\right)=- & \frac{1}{128}\left[(n+1)(n+2)(n+4)(n+5) I_{0, n}^{(3)}\right. \\
& \left.\quad-(n+1)^{2}(n+2)^{2} I_{n+3, n}^{(3)}-12 \sum_{m=1}^{n+2} m\left(n^{2}+6 n+7-2 m^{2}\right) I_{m, n}^{(3)}\right] .
\end{aligned}
$$

Substituting (46) and (77) into the above expression, we encounter again the geometric sum, as well as a sum of the form $\sum_{m} m^{2} x^{m}$. Using (91) and the summation formula [18, Eq. (5.14.9)]

$$
\sum_{m=1}^{n} m^{2} x^{m}=\frac{x(1+x)-x^{n+1}\left[(n+1)^{2}-\left(2 n^{2}+2 n-1\right) x+n^{2} x^{2}\right]}{(1-x)^{3}},
$$

after a tedious but straightforward calculation we arrive at the following closed analytical formula for $E\left(C_{n}^{(3)}\right)$, which is a new result:

$$
\begin{aligned}
E\left(C_{n}^{(3)}\right)= & -\frac{\pi}{128}\left\{2(n+1)(n+2)(n+4)(n+5) \log \left(\frac{(n+1)(n+2)}{2}\right)\right. \\
& +\frac{n^{5}-16 n^{4}-269 n^{3}-1200 n^{2}-2102 n-1250}{n+3} \\
& +\frac{2(n+5)^{2}}{(n+2)(n+3)} \Re\left[\left(\frac{(n+1)(n+5)+\mathrm{i} \sqrt{3(n+1)(n+5)}}{(n+1)(n+2)}\right)^{n+1}\right. \\
& \left.\left.\times\left(2 n^{2}+13 n+14-\mathrm{i}(n+1)(n+6) \sqrt{\frac{(n+1)(n+5)}{3}}\right)\right]\right\} .
\end{aligned}
$$

When $\lambda \geq 4$, combination of (44) and (76) provide an expression for the entropy $E\left(C_{n}^{(\lambda)}\right)$ in terms of finite sums. For the sake of brevity, in (44) it is convenient to absorb the term corresponding to $I_{n+\lambda, n}^{(\lambda)}$ into the sum over $m$ by setting

$$
\beta_{n+\lambda, n}^{(\lambda)}:=-\alpha_{\lambda-1, n}^{(\lambda)} d_{n, n}^{(\lambda)}
$$


instead of using (45) with $m=n+\lambda$, which would give for $\beta_{n+\lambda, n}^{(\lambda)}$ the value $-\alpha_{\lambda-1, n}^{(\lambda)} d_{n, n}^{(\lambda)}+\alpha_{0, n}^{(\lambda)} d_{n+\lambda, n}^{(\lambda)}$. We thus have that

$$
\begin{aligned}
E\left(C_{n}^{(\lambda)}\right)= & -\frac{\pi}{2} c_{n}^{(\lambda)}\left\{2 \alpha_{0, n}^{(\lambda)} d_{0, n}^{(\lambda)} \log \left(\frac{(\lambda)_{n}}{n !}\right)+\alpha_{0, n}^{(\lambda)} d_{n, n}^{(\lambda)}+(2 \lambda-1) \sum_{m=1}^{n+\lambda} \frac{\beta_{m, n}^{(\lambda)}}{m}\right. \\
& -\sum_{m=1}^{n+\lambda} \sum_{k=1}^{m} \sum_{k_{6}=0}^{[m / 3]} \sum_{k_{8}=0}^{[m / 4]} \cdots \sum_{k_{2(\lambda-1)=0}}^{[m /(\lambda-1)]} \beta_{m, n}^{(\lambda)}\left(\frac{\alpha_{\lambda-3, n}^{(\lambda)}}{\alpha_{\lambda-2, n}^{(\lambda)}}\right)^{m}\left(-\frac{\left(\alpha_{\lambda-2, n}^{(\lambda)}\right)^{2}}{\alpha_{\lambda-1, n}^{(\lambda)} \alpha_{\lambda-3, n}^{(\lambda)}}\right)^{k} \\
& \times \frac{(k-1) !}{\left(2 k-m+\sum_{r=1}^{\lambda-3} r k_{2 r+4}\right) !\left(m-k-\sum_{s=1}^{\lambda-3}(s+1) k_{2 s+4}\right) !} \\
& \left.\times \prod_{j=3}^{\lambda-1} \frac{1}{\left(k_{2 j}\right) !}\left(-\frac{\alpha_{\lambda-1-j, n}^{(\lambda)}\left(\alpha_{\lambda-2, n}^{(\lambda)}\right)^{j-2}}{\left(\alpha_{\lambda-3, n}^{(\lambda)}\right)^{j-1}}\right)^{k_{2 j}}\right\}
\end{aligned}
$$

Unlike (20), (97) is completely analytical for all $\lambda \in \mathbb{N}$, which makes it suitable for symbolic computation. For instance, a Maple implementation of the formula enabled us to obtain the closed analytical expressions for $E\left(C_{n}^{(4)}\right)$ and $E\left(C_{n}^{(5)}\right)$, with $1 \leq n \leq 15$, that are displayed in Tables 1 and 2, respectively. In these tables we also provide numerical values of the entropies obtained from the exact ones, in order that the interested reader can compare them with those given by numerical algorithms such as that in [5].

\section{Summary and conclusions}

The problem of obtaining closed analytical formulas for the entropy of orthogonal polynomials is known to be very difficult, as displayed by the fact that in previous work on the subject formulas of this kind were only found for the Gegenbauer polynomials of parameter $\lambda=0,1,2$. Here we have presented a new approach to the calculation of the entropy of Gegenbauer polynomials, based on the use of trigonometric representations for these polynomials, which has allowed us to explicitly evaluate the entropic integrals by means of complex analysis techniques. Using this method we have been able to derive in a unified way closed formulas of $E\left(C_{n}^{(\lambda)}\right)$ for $\lambda=1,2,3$, the last one being new. Furthermore, when $\lambda \geq 4, \lambda \in \mathbb{N}$, we have obtained completely analytical expressions of the entropy in terms of finite sums, which easily provide exact values for the entropy using symbolic computation. The growing complexity in the formulas of $E\left(C_{n}^{(\lambda)}\right)$ as $\lambda$ increases serves as a clear illustration of the difficulties posed by the calculation of the entropy of orthogonal polynomials.

When the parameter $\lambda$ is not a positive integer, the Szegö representation (27) of the Gegenbauer polynomial $C_{n}^{(\lambda)}$ has infinitely many terms, so the same happens for the expressions (35) and (41) of the entropic integral $E\left(C_{n}^{(\lambda)}\right)$. It remains open the problem of studying the convergence behaviour of these series, as well as that of summing up them analytically. It would be of particular interest to obtain exact analytical expressions for the entropy of Gegenbauer polynomials of half-integer parameter since, as already 
Table 1. Exact and numerical values of the entropy $E\left(C_{n}^{(4)}\right)$ for $1 \leq n \leq 15$.

\begin{tabular}{|c|c|c|}
\hline $\mathrm{n}$ & Exact value & Numerical value \\
\hline 1 & $-7 \pi \log (2)+\frac{119}{240} \pi$ & -13.685 \\
\hline 2 & $-\frac{105}{8} \pi \log (10)+\frac{580771}{300000} \pi$ & -88.862 \\
\hline 3 & $-\frac{75}{2} \pi \log (20)+\frac{95}{21} \pi$ & -338.714 \\
\hline 4 & $-\frac{5775}{64} \pi \log (35)+\frac{4883222845}{632481024} \pi$ & -983.613 \\
\hline 5 & $-\frac{385}{2} \pi \log (56)+\frac{17355685}{1806336} \pi$ & -2404.173 \\
\hline 6 & $-\frac{3003}{8} \pi \log (84)+\frac{6449434961}{1058158080} \pi$ & -5206.005 \\
\hline 7 & $-\frac{1365}{2} \pi \log (120)-\frac{1396715852287}{139218750000} \pi$ & -10296.556 \\
\hline 8 & $-\frac{75075}{64} \pi \log (165)-\frac{24757176334716125}{493018566815808} \pi$ & -18974.368 \\
\hline 9 & $-1925 \pi \log (220)-\frac{1200329915}{9135984} \pi$ & -33031.075 \\
\hline 10 & $-\frac{12155}{4} \pi \log (286)-\frac{325291539600149215255}{1172732412725203616} \pi$ & -54866.421 \\
\hline 11 & $-4641 \pi \log (364)-\frac{31458443588344487293819}{60436675052957701680} \pi$ & -87616.538 \\
\hline 12 & $\frac{440895}{64} \pi \log (455)-\frac{25537984326378849719971131}{28270687046875000000000} \pi$ & -135295.739 \\
\hline 13 & $-9975 \pi \log (560)-\frac{1779685691911133495}{1202109806542848} \pi$ & -202952.031 \\
\hline 14 & $-\frac{56525}{4} \pi \log (680)-\frac{36234350694889865223938313068785}{15613637127259094259005915136} \pi$ & -296836.555 \\
\hline 15 & $-19635 \pi \log (816)-\frac{130243656594168370141034405}{37115886521993021558784} \pi$ & -424587.139 \\
\hline
\end{tabular}

mentioned in Sec. 1, they are needed together with those of the integer case in order to evaluate the information entropy of spherical and hyperspherical harmonics. Finally, it would also be desirable to extend the method introduced in this paper to other families of orthogonal polynomials having trigonometric representations, a line of research that is currently being developed. 
Table 2. Exact and numerical values of the entropy $E\left(C_{n}^{(5)}\right)$ for $1 \leq n \leq 15$.

\begin{tabular}{|c|c|c|}
\hline $\mathrm{n}$ & Exact value & Numerical value \\
\hline 1 & $-\frac{525}{128} \pi \log (5)+\frac{945}{1024} \pi$ & -17.839 \\
\hline 2 & $-\frac{2475}{128} \pi \log (15)+\frac{27685925}{5225472} \pi$ & -147.857 \\
\hline 3 & $-\frac{17325}{256} \pi \log (35)+\frac{61634724075}{3373232128} \pi$ & -698.499 \\
\hline 4 & $-\frac{25025}{128} \pi \log (70)+\frac{5573831525}{115605504} \pi$ & -2457.981 \\
\hline 5 & $-\frac{63063}{128} \pi \log (126)+\frac{338107973281463}{3173748645888} \pi$ & -7150.909 \\
\hline 6 & $-\frac{143325}{128} \pi \log (210)+\frac{20887195}{101376} \pi$ & -18162.369 \\
\hline 7 & $-\frac{75075}{32} \pi \log (330)+\frac{1408430247274269205}{3944148534526464} \pi$ & -41620.201 \\
\hline 8 & $-\frac{294525}{64} \pi \log (495)+\frac{806559968327725}{1438588584576} \pi$ & -87940.792 \\
\hline 9 & $-\frac{546975}{64} \pi \log (715)+\frac{29915266041851863399425}{37527437207206515712} \pi$ & -173958.634 \\
\hline 10 & $-\frac{969969}{64} \pi \log (1001)+\frac{97664804776687286561309}{96698680084732322688} \pi$ & -325775.232 \\
\hline 11 & $-\frac{6613425}{256} \pi \log (1365)+\frac{230209361727271224010045}{212679240849405517824} \pi$ & -582478.486 \\
\hline 12 & $-\frac{2723175}{64} \pi \log (1820)+\frac{10188450005911283085}{12635587626401792} \pi$ & -1000899.539 \\
\hline 13 & $-\frac{4352425}{64} \pi \log (2380)-\frac{39663465263970548089202600252605}{249818194036145508144094642176} \pi$ & -1661590.212 \\
\hline 14 & $-\frac{6774075}{64} \pi \log (3060)-\frac{717231543067734581588054629334401175}{306761704661739640893688309874688} \pi$ & -2676220.464 \\
\hline 15 & $-\frac{5148297}{32} \pi \log (3876)-\frac{535111116210266542852402527915814650511}{82233794352493419438828330115762176} \pi$ & -4196611.889 \\
\hline
\end{tabular}

\section{Acknowledgments}

The second author (S. Gandy) gratefully acknowledges the hospitality of the Departamento de Matemáticas of the Universidad Carlos III de Madrid, where this research was carried out, as well as financial support from the European Union Socrates/Erasmus Programme. The work of the first and third authors (J.I. de Vicente and J. Sánchez-Ruiz) was supported by Universidad Carlos III de Madrid, Comunidad 
Autónoma de Madrid (project No. UC3M-MTM-05-033), and Dirección General de Investigación (MEC) of Spain under grant MTM2006-13000-C03-02. The work of the third author was also supported by the Dirección General de Investigación (MEC) of Spain grant FIS2005-00973, and the Junta de Andalucía research group FQM-0207.

\section{References}

[1] Shannon C E 1948 A mathematical theory of communication Bell Syst. Tech. J. 27 379-423, 623-56 Reprinted in Shannon C E and Weaver W (ed) 1949 The Mathematical Theory of Communication (Urbana, IL: University of Illinois Press)

[2] Beckner W 1975 Inequalities in Fourier analysis Ann. Math. 102 159-82

[3] Bialynicki-Birula I and Mycielski J 1975 Uncertainty relations for information entropy in wave mechanics Commun. Math. Phys. 44 129-32

[4] Dehesa J S, Martínez-Finkelshtein A and Sánchez-Ruiz J 2001 Quantum information entropies and orthogonal polynomials J. Comput. Appl. Math. 133 23-46

[5] Buyarov V S, Dehesa J S, Martínez-Finkelshtein A and Sánchez-Lara J 2004 Computation of the entropy of polynomials orthogonal on an interval SIAM J. Sci. Comput. 26 488-509

[6] Szegó G 1975 Orthogonal Polynomials (Am. Math. Soc. Colloq. Publ. vol 23) 4th edn (Providence, RI: American Mathematical Society)

[7] Yáñez R J, Van Assche W and Dehesa J S 1994 Position and momentum information entropies of the D-dimensional harmonic oscillator and hydrogen atom Phys. Rev. A 50 3065-79

[8] Dehesa J S, Van Assche W and Yáñez R J 1997 Information entropy of classical orthogonal polynomials and their application to the harmonic oscillator and Coulomb potentials Meth. Appl. Anal. 4 91-110

[9] Yáñez R J, Van Assche W, González-Férez R and Dehesa J S 1999 Entropic integrals of hyperspherical harmonics and spatial entropy of $D$-dimensional central potentials $J$. Math. Phys. $405675-86$

[10] Buyarov V S 1997 On information entropy of Gegenbauer polynomials Vestn. Mosk. Univ. Ser. 1 Mat. Mekh. 6 8-11 (in Russian)

[11] Buyarov V S, López-Artés P, Martínez-Finkelshtein A and Van Assche W 2000 Information entropy of Gegenbauer polynomials J. Phys. A: Math. Gen. 33 6549-60

[12] Sánchez-Ruiz J 2003 Information entropy of Gegenbauer polynomials and Gaussian quadrature $J$. Phys. A: Math. Gen. 36 4857-66

[13] Sánchez-Lara J and Sánchez-Ruiz J 2005 Information entropy of the Jacobi polynomials $P_{n}^{(m-1 / 2, \pm 1 / 2)}$ Preprint (unpublished)

[14] Andrews G E, Askey R and Roy R 1999 Special Functions (Cambridge: Cambridge University Press)

[15] Szegő G 1934 Über gewisse orthogonale polynome, die zu einer oszillierenden Belegungsfunktion gehören Math. Ann. 110 501-13 (in German)

[16] Roman S 1980 Faà di Bruno's formula Amer. Math. Monthly 87 805-9

[17] Graham R L, Knuth D E and Patashnik O 1994 Concrete Mathematics (Reading, MA: AddisonWesley)

[18] Hansen E R 1975 A Table of Series and Products (Englewood Cliffs, NJ: Prentice-Hall) 